\title{
An Artificial Management Platform Based on Deep Learning Using Cloud Computing for Smart Cities
}

\author{
Yunus Santur ${ }^{1}$, Ebru Karaköse ${ }^{2}$, Mehmet Karaköse ${ }^{1}$, Erhan Akın $^{1}$
}

Accepted : 12/05/2017 Published: 21/08/2017

DOI: 10.18100/ijamec.2017Special Issue30466

Abstract: In today's world; smart city management uses sensors, cameras and mobile devices which is internet-connected. These devices, called Internet of things (IoTs) generate large amounts of data. A big data-based approach is needed to store these data appropriately and to provide real-time access to the data. In smart city management applications that use many different sources of information, traditional machine learning methods for classifying large data and generating meaning can be inadequate. Deep learning approach is widely used today to solve similar problems. In this study, a cloud computing based architectural approach is proposed that enables data mining using deep learning on big data produced by IOTs.

Keywords: Big Data, Cloud Computing, Deep Learning, Deep Mining, IoT, Smart City

\section{Introduction}

Smart city is a concept based on the principle of using technological infrastructure in city management for the purposes of management, planning, analysis, improving the quality of service new services [1]. In this sense, smart cities include smart management, smart transportation, smart technologies, smart economy, smart health and so on, as in "Fig. 1".

In today's world, many countries are producing new projects for smart cities in metropolitan areas and allocating huge budgets for this purpose. This is the biggest disadvantage of smart cities as well as their advantages [2]. In smart city applications, it is necessary to collect, evaluate and analyze the data to be obtained from many sources of information and sensors and to interpret the results, and the actions need to be achieved [3]. The action to be achieved can be cost reduction, taking new managerial decisions, improving the quality of service and so on.

For instance, smart traffic application gives real-time road information for the drivers by means of mobile applications. This information may indicate real-time situations such as instant traffic density on the route, if any, maintenance work and accident. The input data of the system to be created for this purpose consist of traffic lights, city surveillance and cameras. The system has outputs such as informing, dynamic operation of traffic lights and directions [4].

Smart city applications generally require algorithms with different types of data inputs and outputs that will perform realtime learning on this big data. Traditional machine learning methods are not sufficient for smart city applications in terms of processing power and memory consumption.

\footnotetext{
${ }^{1}$ Computer Engineering Department, Engineering Faculty, Furat University, Campus, 23100, Elazığ/Turkey

${ }^{2}$ Civil Aviation High School, Firat University, Elazığ/Turkey

* Corresponding Author: Email: ysantur@firat.edu.tr

Note: This paper has been presented at the 5th International Conference on Advanced Technology \& Sciences (ICAT'17) held in Istanbul (Turkey), May 09-12, 2017.
}

Deep Learning (DP) is successfully applied in image recognition, object tracking, analysis and interpretation applications on big and multilayer data $[5,6]$.

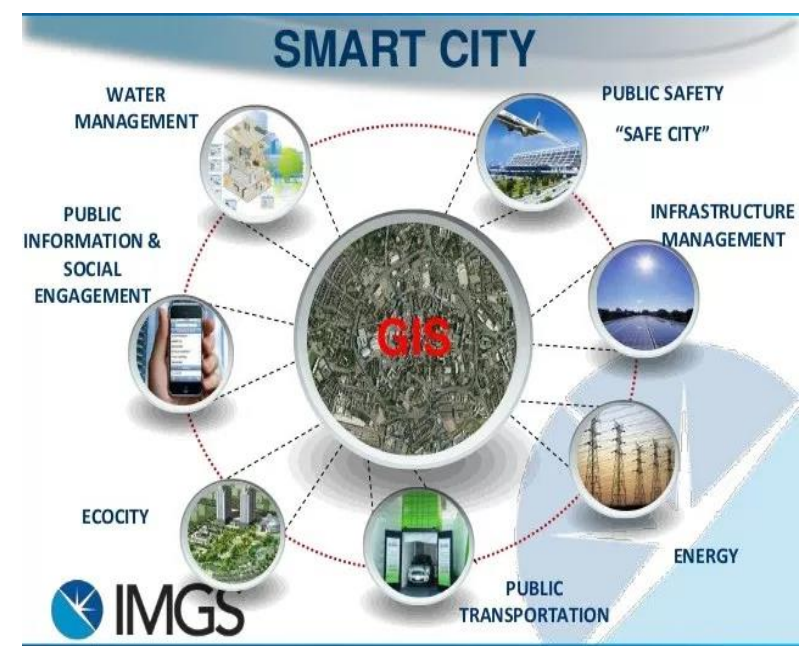

Fig.1. Smart city applications [5]

Smart city applications, as in this example, generally require algorithms with different types of data inputs and outputs that will perform real-time learning on this big data. Traditional machine learning methods are not sufficient for smart city applications in terms of processing power and memory consumption. Deep Learning (DP) is successfully applied in image recognition, object tracking, analysis and interpretation applications on big and multilayer data [5,6].

Deep Belief Networks, Convolutional Neural Networks (CNNs) and Deep Boltzmann Machines are widely used for DP applications in the literature. However, CNNs are more commonly used along with their easier training, need for fewer parameters, ease of implementation and success rates compared to other methods. 
The biggest reasons for the popularity of deep learning networks after 2011 are the fact that learning process is simpler and that the costs of graphics process unit (GPU) are at very reasonable levels compared to other machine learning algorithms. Deep learning algorithms working on GPUs provide speed increase by tens of times compared to traditional Central Programming Units (CPUs) depending on GPU properties $[8,9]$.

Apart from PC and mobile devices, various sensors and electronic appliances have structures connected to internet via wifi or mobile networks. Nowadays, the number of these devices, that are called internet of things (IoT), has exceeded 1.2 billion [10]. IoTs collect information such as moisture, heat, carbon dioxide ratio, motion, speed, etc. through the sensors they have. Thus, IoTs are widely used in remote and real-time applications such as smart building management, smart parking and smart monitoring. Even world's giant companies like Google are producing development cards and cloud-based software solutions for IoT, and IoTs are expected to have a greater importance in our lives in the near future [11].

When the number of IoTs used in distributed architecture for different purposes, the amount of data they produce and the rate of data generation are taken into account, the resulting data type is considered as big data [12]. It is necessary to store these data by modeling with a suitable architecture for processing, to clear inconsistent and unnecessary data and to perform dimension reduction before processing the data. A Big data which is appropriate for this structure is usually not regular and consistent, therefore it cannot be stored as sql-based. For this purpose, it should mainly be stored in a cloud-based and distributed structure with a nosql -based database [13].

Big data is mentioned by the concepts called $5 \mathrm{~V}$ in the literature. These $5 \mathrm{~V}$ concepts can be associated with smart cities and IoTs as the following.

Variety: It refers to variety of data. Data is not hierarchical and uniform when IoTs that include image, video, automation sources and many sensors as input data in smart city applications are taken into account.

Velocity and Volume: It refers to the growth rate and size of data. More than 1.2 billion IoT's worldwide, more than 200 million cameras that are connected to internet are recording 1.4 trillion hours video per year [8].

Verification: The data obtained from many different sources do not have a hierarchical and homogeneous structure. It is necessary to filter out the data that are unnecessary or disturb the hierarchical structure for learning from data.

Value: It refers to the value created by the data. It is the value created by the output which is obtained as a result of data analysis. A new smart city application, ensuring cost in the management of city resources and improving the quality of services offered to the public can be as examples.

When the literature on smart city applications is examined, two major technologies, IoTs and Big Data, come to the forefront.

Paganelli et al. defined a web architecture for accessing remote IoTs to be used in smart cities. In the study, each IoT was represented by a unique identity. Along with the Restful-based web architecture, the data from these IoTs were accessed via json apis [13].

Dlodlo et al. defined the cloud-based data storage architecture by giving information about IoT development environments such as Aurdino and Raspery Pi for smart applications to be used in smart cities [15].
Shah and Misra implemented a mobile application that performs remote monitoring by reading the environmental variables such as moisture, temperature and $\mathrm{CO} 2$ in the environment through IoTs for smart environmental monitoring application with the aim of reducing air pollution [16].

Sakhardande et al. proposed a structure that uses more than one IOT network with power supply and wifi network in order to perform monitoring even in disastrous situations in smart cities [17].

Costa and Santos defined architecture for the use of Big Data in smart cities. In the study called Basis, they presented an architecture that uses hdfs in which Big Data is stored with cloudbased hadop technology [14].

Horban proposed an architecture that performs data mining on Big Data for smart energy management by detailing the relationship between the concept of Big Data and smart cities [18].

Alshawish et al. proposed an architecture for the use of Big Data in smart city applications. The prominent suggestion in the study is the 6-step, reusable Big Data pipeline architecture for smart management [19].

Table 1. Literature Review

\begin{tabular}{|c|c|c|}
\hline $\begin{array}{l}\text { Major } \\
\text { Concept }\end{array}$ & Brief Summary & Reference \\
\hline $\begin{array}{l}\text { Deep } \\
\text { Learning }\end{array}$ & $\begin{array}{l}\text { Survey study related to Deep Learning } \\
\text { algorithms }\end{array}$ & [7] \\
\hline IoTs & $\begin{array}{l}\text { Restfull framework for IOTs to be used in } \\
\text { Smart City }\end{array}$ & [13] \\
\hline IoTs & $\begin{array}{l}\text { Research on Iots platforms that can be } \\
\text { used in Smart cities }\end{array}$ & [15] \\
\hline IoTs & $\begin{array}{l}\text { IoTs-based approach for smart city's } \\
\text { condition monitoring }\end{array}$ & [16] \\
\hline IoTs & $\begin{array}{l}\text { IoTs-based framework for smart city } \\
\text { disaster management }\end{array}$ & [17] \\
\hline Big Data & Big Data structure for smart cities & [14] \\
\hline Big Data & $\begin{array}{l}\text { Data mining analysis on Big Data for } \\
\text { smart energy use }\end{array}$ & {$[18]$} \\
\hline Big Data & Big Data applications in smart cities & [19] \\
\hline
\end{tabular}

Two major technologies, Big Data and IoT, come to the forefront in smart city applications in the literature examples summarized in Table-1. However, when today's needs for smart city applications are taken into account, it is thought that Deep Learning will be the most appropriate machine learning on Big Data from IoTs for the processes of real-time action decisions, analysis and acquisition of valuable information.

In addition to these two major technologies, the proposed system needs to use a cloud-based distributed architecture to handle large data and produce results.

In this study, an architecture using Deep Learning was proposed for valuable knowledge mining in smart city applications.

\section{Proposed Approach}

In this study, an approach based on Deep Learning that uses CNN on Big Data coming from all IoTs was proposed for smart city management. The study involves the steps given in "Fig.2". A unique id that will replace the identity is given for each IoT. This is necessary to obtain the type, location, sensors and values of the relevant IoT [13] in a restful architecture as in the study. 
The collected data should be stored in a distributed architecture in accordance with the Big Data architecture on a nosql-based cloud server. In Big Data architecture, data should be stored on distributed server clusters and be subjected to map-reduce process without processing [20]. The outputs obtained after the mapreduce processing will create the entries for the Deep Learning training process.

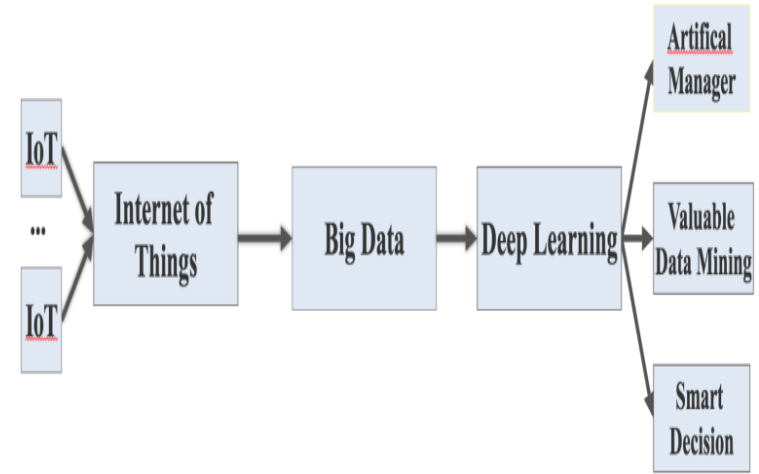

Fig.2. Block diagram of the proposed approach

\subsection{Internet of Things (IoTs)}

Electronic devices such as cameras, sensors and measuring devices are regarded as Things provided that they are connected to at least one sensor, a unique identity and internet. A device that fulfills these three conditions is accessible from all over the world, so it is manageable. Nowadays, IoT platform is provided with many corporate development cards and hardware and software supports. IoT developer kit for Google cloud-supported platform is seen in "Fig.3" [21]. The developer kit has sensors such as wifi, acclerometer, temperature, light, rotary and distance and is ready for use via google cloud platform.

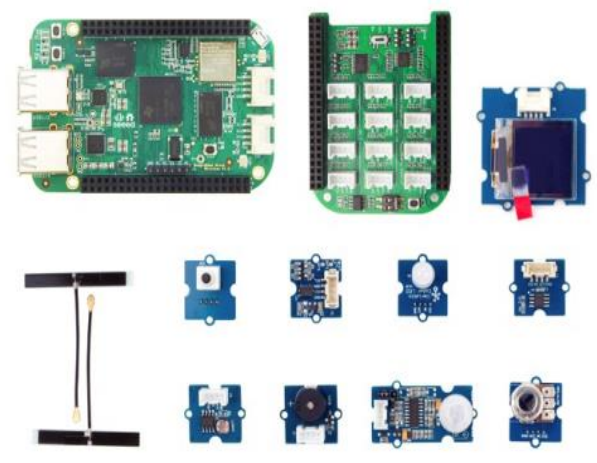

Fig.3. Google cloud supported IoT developer kit [21]

\subsection{Big Data}

When the fact that there are 1.2 billion IoT around the world is taken into account, Big Data is the type of data that occurs in terms of the variety, rate, size of the produced data and the value created by it. The basic properties that should be known about Big Data are explained below in accordance with the Big Data life cycle is seen in "Fig.4".

Hadoop Distributed File System (HDFS): It is the file system consisting of distributed server clusters for Big Data.

Map: Map-Reduce processing was announced by Google in 2004 [23]. In the map phase, the data received from the host node is divided into smaller segments and distributed to the child nodes.

Reduce: In this phase, concrete analysis results are obtained on the data obtained in the map phase.
Hadoop: It is a project that performs Map-Reduce process on Distributed File System [24].

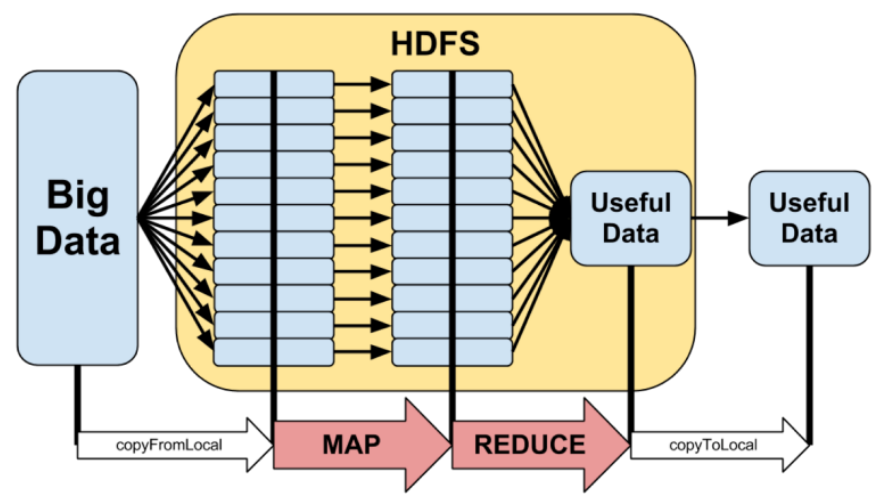

Fig.4. Big data map-reduce process [22]

\subsection{Cloud Computing}

All client service providers for today's big data-processing web applications. It uses cloud based technology to meet both scenarios such as disaster recovery and simultaneous service with all clients. The major advantages of cloud-based technology, shown in "Fig.5", are listed below [17,21,24].

- Diseaster Recovery: One or more of the cloud services provide continuous protection, even if it is inoperable [17].

- Load Balancing: Provides load balancing for all clients (monitoring) or IoTs interacting with the system.

- Distributed Computing: Data distributes the load on the appropriate services. The incoming service meets the most appropriate service.

- Backup: It provides affordable solution for backup and big data.

- Performance: Adding and removing new nodes to the network can be done easily depending on the performance required.

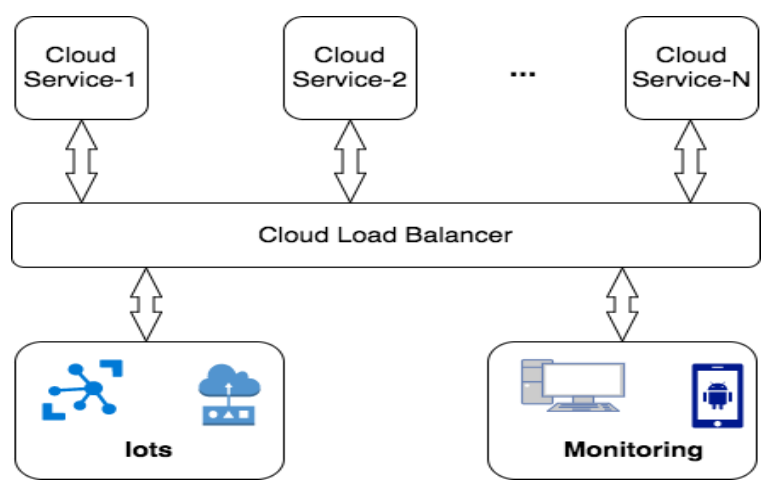

Fig.5. Cloud load balancer

\subsection{Deep Learning}

Deep Learning, which is a machine learning method and is a special form of Artifical Neural Networks (ANN) is successfully applied in applications such as information retrieval [25], image recognition, object tracking and language processing [26,27].

CNNs are preferred for the reasons such as the simplicity of its training process, fast running in test phase and ease of application among Deep Learning Algorithms. In general, CNNs consist of 5 stages. 
Input Layer: It creates the input data of the system. In the proposed method, the outputs of Big Data Map-Reduce process will create the CNN entry for smart city management. The use of outputs after Map-Reduce process in Deep Learning training will ensure that the training data does not contain any inconsistencies and that a model that characterizes the learning model very well will emerge.

Convolutional Layer: It is also known as subsampling. It is the process of subsampling by taking a core matrix of smaller size on input data as it is given in " 1 ".

$C_{i j}^{l}=\sum_{a=0}^{m-1} \sum_{b=0}^{m-1} w_{a b} f_{(i+a)(j+b)}^{l-1}$

Pooling Layer: It is the feature extraction step. Feature selection is performed on the entries multiplexed in the previous step. A kernel matrix is also used while performing feature selection. For instance, In the event that $2 \times 2$ kernel matrix is used, max, min or mean value is selected as feature from a total of 4 values. Thus, a feature vector is obtained as given in "2,3".

$P_{\text {vec }}=\sum_{i} C_{i}{ }^{*} \mathrm{p}$

$\sum_{1}^{n} P_{v e c}=\{\min |\max | \operatorname{mean}\} \sum_{i} C_{i}$

Connected Layer: It is the last layer before output layer. Convolutional and Pooling layers in CNNs can be recursively selected in desired number. It is important that underfitting and overfitting do not occur on the network. One of the methods that can be used for this purpose is the Mean Square Error (MSE) analysis given in "4".

$M S E=\frac{1}{n} \sum_{1}^{n}\left(Y_{i}-Y\right)^{2}$

Outputs: It is the output layer as the number of classifiers. System output can be linear classifier or predict rate for a class depending on the learning model.

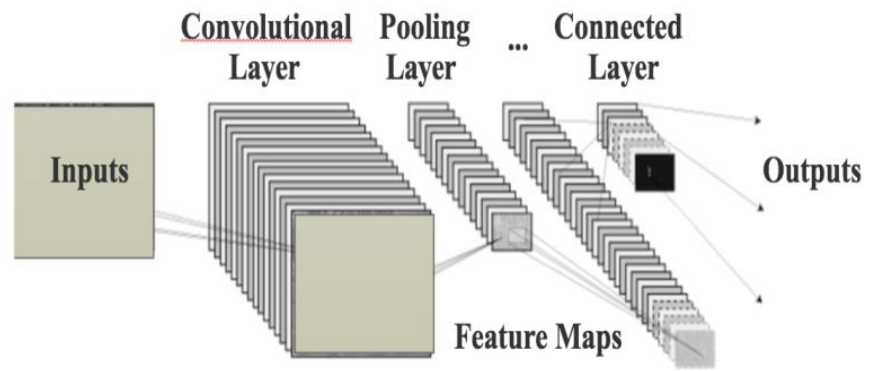

a) Deep learning with $\mathrm{CNN}$ for smart cities
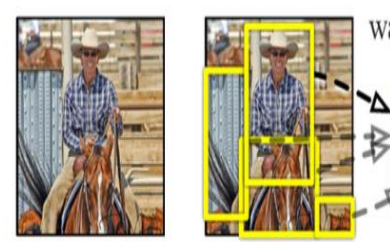

warped region

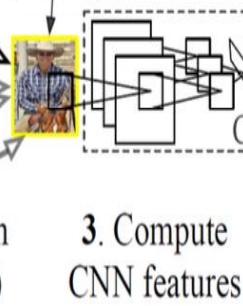

aeroplane? no.

\begin{abstract}
1. Input
image
\end{abstract}

2. Extract region
CNN features
In "Fig.6", the illustration of the study conducted by Girshick et al. is presented for a typical $\mathrm{CNN}$ and scene interpretation, objet recognition and semantic segmentation [28-30].

Running Deep Learning applications on a traditional CPU is a disadvantage in terms of training process, and it is not possible to use it in real time systems.

For this purpose, Nvidia display card manufacturer has developed parallel programming framework Cuda library on display card for Deep Learning. To perform Deep Learning applications by GPU programming on Nvidia GPU shows great similarity with CPU programming. In this way, the lack of learning cost of parallel/distributed programming for Deep Learning is a great advantage.

A figure that compares CPU and GPU environments for Deep Learning is presented in "Fig.7". While the compared Intel 6th Generation has i5 4 cores, Nvidia Geforce 930mx gpu has 384 cores. Nowadays, CPUs can have 32 and GPUs can have 3584 (Nvidia Titan X) cores.

GPUs are the reasons for these architectures and provide speed increase at least by tens of times in Deep Learning applications compared to CPUs. It is possible to increase this rate further by using a grid of GPUs.

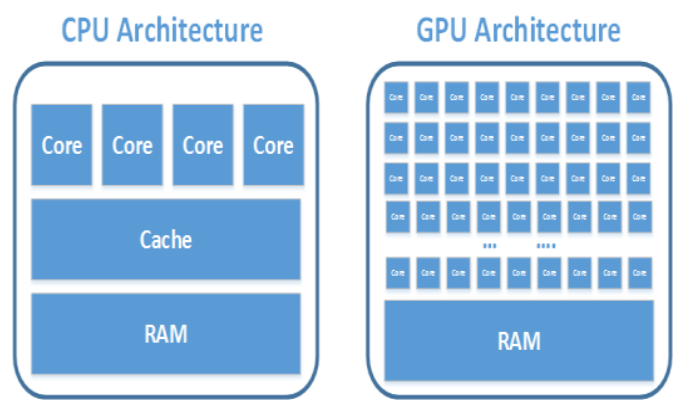

Fig.7. CNN for deep learning

\section{Conclusions}

Smart cities is a whole of concepts in which smart applications are used for the purposes of the management of city resources, improving the quality of service, obtaining valuable information that will affect managerial decisions and reducing costs. It consists of sub-elements such as smart traffic, smart health, smart building, smart monitoring and smart infrastructure.

IoTs are the source of information in smart city applications. The fact that they are manageable due to the sensors they have and their internet connection provide unmanned source of information in smart city applications. However, both the number of IoT, the amount of data produced and the rate of data generation require the use of a Big Data-based structure for IoTs.

Traditional machine learning algorithms will be inadequate in processing this data in terms of workload and working speeds in order to perform data mining, supervised/unsupervised learning in such applications where there is a large number of data input and the data type is Big Data.

Especially after 2011, Deep Learning has been successfully applied in many applications such as object recognition, scene interpretation, language processing and driverless tools for the reasons of reduced GPU costs, working speeds, ease of implementation.

In this study, a deep learning based approach was proposed with the purpose of performing artifical management in smart cities where IoTs constitute information resources.

Fig.6. CNN for deep learning 
The proposed approach has four steps. It was projected that the raw data received from IoTs are stored in a distributed architecture in accordance with the Big Data architecture in the first step, that a consistent and well-modeled data set to be used in the training process is obtained by performing Map and Reduce process on Big Data in the second step, and that a CNNbased Deep Learning training is performed in the third step.

The final step is that the whole system works in a distributed cloud architecture. Incoming requests are met by the load balancer and the most appropriate node is forwarded and processed. This approach provides for expandable, modular and parallel operation.

Thus, in this study, an approach using deep learning was proposed with the aim of obtaining valuable information by performing data mining in a smart city management.

\section{Acknowledgements}

This paper presented in 5th International Conference on Advanced Technology \& Sciences (ICAT'17)

\section{References}

[1] H. Chourabi, T. Nam, S. Walker, J.R. Gil-Garcia, S. Mellouli, K. Nahon, H.J. Scholl, "Understanding smart cities: An integrative framework", In System Science (HICSS), 2012 45th Hawaii International Conference on (pp. 2289-2297), 2012.

[2] T. Nam, T. A. Pardo, "Conceptualizing smart city with dimensions of technology, people, and institutions", In Proceedings of the 12th Annual International Digital Government Research Conf.: Digital Government Innovation in Challenging Times (pp. 282-291), 2012.

[3] K. Su, J. Li, H. Fu, "Smart city and the applications", In Electronics, Communications and Control (ICECC), 2011 International Conference on (pp. 1028-1031), 2011.

[4] C.T. Barba, M. A. Mateos, P. R. Soto, A. M. Mezher, M. A. Igartua, "Smart city for VANETs using warning messages, traffic statistics and intelligent traffic lights", In Intelligent Vehicles Symposium (IV), (pp. 902-907), 2012.

[5] Online (2017), http://joshconsult.com/2017/07/05/applicationgeographic-information-system-gis-smart-cities/

[6] Y. Santur, M. Karaköse, E. Akın, "Learning Based Experimental Approach for Condition Monitoring Using Laser Cameras In Railway Tracks", International Journal of Applied Mathematics, Electronics and Computers (IJAMEC), 4, pp.1-5, 2016.

[7] L. Wang, D. Sng, "Deep Learning Algorithms with Applications to Video Analytics for A Smart City: A Survey", arXiv preprint arXiv:1512.03131, 2015.

[8] P.Gupta,http://www.nvidia.com.tw/content/PDF/GTC/2015/sm artcity/gpu-accelerated-platform-pradeep-gupta.pdf, 2015.

[9] Y. Santur, M. Karaköse, E. Akin, "Improving of personal educational content using big data approach for mooc in higher education", In Information Technology Based Higher Education and Training (ITHET), 2016 15th International Conference on (pp. 1-4), 2016.

[10] N. Dlodlo, O. Gcaba, A. Smith,'Internet of things technologies in smart cities", In IST-Africa Week Conference, (pp. 1-7), 2016.

[11]F. Paganelli, S. Turchi, D. Giuli, "A web of things framework for restful applications and its experimentation in a smart city", 2014.
[12]R. Kitchin, "The real-time city? Big data and smart urbanism", GeoJournal, 79(1), 1-14, 2014.

[13]F. Paganelli, S. Turchi, D. Giuli, "a web of things framework for restful applications and its experimentation in a smart city", 2014.

[14]C. Costa, M.Y. Santos, "BASIS: A big data architecture for smart cities", In SAI Computing Conference (SAI), 2016 (pp. 1247-1256), 2016.

[15] N. Dlodlo, O. Gcaba, A. Smith, "Internet of things technologies in smart cities", In IST-Africa Week Conference, pp. 1-7, 2016.

[16]J. Shah, B. Mishra, "IoT enabled environmental monitoring system for smart cities", In Internet of Things and Applications (IOTA), International Conference on (pp. 383-388), 2016.

[17]P. Sakhardande, S. Hanagal, S. Kulkarni, "Design of disaster management system using IoT based interconnected network with smart city monitoring", In Internet of Things and Applications (IOTA), International Conference on (pp. 185190), 2016.

[18]V. Horban, "A multifaceted approach to smart energy city concept through using big data analytics", In Data Stream Mining \& Processing (DSMP), IEEE 1.Int. Conf. on (pp. 392396), 2016.

[19]R.A Alshawish, S. A., Alfagih, M. S. Musbah, "Big data applications in smart cities", In Engineering \& MIS (ICEMIS), International Conference on (pp. 1-7), 2016.

[20] J. Dittrich, J.A. Quiané-Ruiz, "Efficient big data processing in Hadoop MapReduce", Proceedings of the VLDB Endowment, 5(12), 2014-2015.

[21] Online (2016), https://cloud.google.com/solutions/iot/kit/

[22] Online (2016), http://www.glennklockwood.com/dataintensive/hadoop/overview.html

[23] Online (2016), www.google.com

[24]T. White, "Hadoop: The definitive guide", O'Reilly Media, Inc." ,2012.

[25]Y. Santur, S. G. Santur, M. Karaköse, "Knowledge Mining Approaach For Healthy Monitoring from Pregnancy Data with Big Volumes", International Journal of Intelligent Systems and Applications in Engineering (IJISAE), 4, 141-145, 2016.

[26] Y. Santur, M. Karakose, E. Akin, "Random Forest Based Diagnosis Approach for Rail Fault Inspection in Railways", International Conference on Electrical and Electronics Engineering (Eleco 2015), 9.th, pp.714-719, 2015.

[27]Y. Santur, M. Karaköse, E. Akın, "Condition Monitoring Approach Using 3d Modelling of Railway Tracks with Laser Cameras", 4th International Conference on Advanced Technology \& Sciences, pp.132-135, 2016.

[28]R. Girshick, J. Donahue, T. Darrell, J. Malik, "Rich feature hierarchies for accurate object detection and semantic segmentation", In Proceedings of the IEEE conference on computer vision and pattern recognition (pp. 580-587), 2014.

[29] Y. Santur, M. Karaköse, E. Akın, "Chouqet Fuzzy Integral Based Condition Monitoring and Analysis Approach Using Simulation Framework for Rail Faults", 14 ${ }^{\text {th }}$ International Conference on Industrial Informatics (INDIN), 2016 International Conference on (pp. 345-350), 2016.

[30] İ. Aydın, M. Karaköse, E. Akın, "Combined Intelligent Methods Based on Wireless Sensor Networks for Condition Monitoring and Fault Diagnosis", Journal of Intelligent Manufacturing, 26(4), 717-729, 2015. 